\title{
Reflets
}

Revue ontaroise d'intervention sociale et communautaire

\section{L'évolution du travail social : une histoire à suivre - Entrevue avec Roland Lecomte}

\section{Madeleine Dubois et Marie-Luce Garceau}

Volume 6, numéro 1, printemps 2000

Approches d'intervention : définir et renouveler nos pratiques

URI : https://id.erudit.org/iderudit/026293ar

DOI : https://doi.org/10.7202/026293ar

Aller au sommaire du numéro

Éditeur(s)

Reflets : Revue ontaroise d'intervention sociale et communautaire

ISSN

1203-4576 (imprimé)

1712-8498 (numérique)

Découvrir la revue

Citer ce document

Dubois, M. \& Garceau, M.-L. (2000). L'évolution du travail social : une histoire à suivre - Entrevue avec Roland Lecomte. Reflets, 6(1), 18-34.

https://doi.org/10.7202/026293ar

Tous droits réservés (C) Reflets : Revue ontaroise d'intervention sociale et communautaire, 2000
Ce document est protégé par la loi sur le droit d'auteur. L'utilisation des services d'Érudit (y compris la reproduction) est assujettie à sa politique d'utilisation que vous pouvez consulter en ligne.

https://apropos.erudit.org/fr/usagers/politique-dutilisation/ 


\section{L'évolution du travail social : une histoire à suivre}

La présentation d'un numéro portant sur l'analyse des pratiques invite une certaine réflexion sur l'évolution de la pensée et des théories qui ont contribué à la transformation de ces pratiques et ce, dans des contextes socio-politiques particuliers. Pour donner une saveur à la fois très vivante et réflexive à ce bilan de l'évolution du travail social depuis la fin des années 1950 jusqu'à maintenant, nous avons demandé à Roland Lecomte, directeur-fondateur et professeur à l'École de service social de l'Université d'Ottawa, de partager ses souvenirs et de nous faire bénéficier de la richesse de ses expériences.

L'entrevue que nous vous présentons s'est déroulée très amicalement le 10 février 2000. Elle a été dirigée par Madeleine Dubois et Marie-Luce Garceau. Toutes deux avons largement bénéficié des propos de Roland Lecomte et l'entrevue nous a permis de faire le point sur diverses facettes de notre propre histoire de travailleuses sociales. Il ne nous reste qu'à espérer qu'elle vous permettra aussi de faire un court voyage dans le temps, de faire le point, d'ouvrir d'autres avenues au questionnement de nos pratiques pour le bénéfice des personnes que nous servons.

Reflets: Pouvez-vous nous parler des étapes marquantes qui ont façonné votre propre trajectoire comme travailleur social depuis la fin les années 1950.

R. Lecomte: Il est fascinant d'étudier l'itinéraire professionnel d'un individu en comparaison avec celui de la profession à laquelle il appartient. La profession est jeune, moi aussi... La première constatation qui me vient à l'esprit est l'évolution rapide et marquante de la formation en service social. Lorsque j'ai fait mes études en service social en 1960, le baccalauréat n'existait pas. Jusqu'en 1967, la formation en travail social ne s'offrait qu'au niveau de la maîtrise, 
et ce, partout au Canada et en Amérique du Nord. À cette époque, l'influence de la religion était très grande et la présence de l'État était moins marquante. Le Collège St-Patrick's, où j'ai fait mes études, était affilié avec l'Université d'Ottawa. Le programme de maitrise ne se donnait qu'en anglais par des pères Oblats que l'on qualifiait «d'Oblats protestants» parce qu'ils ne parlaient pas français... Les cours étaient essentiellement basés sur une approche néo-freudienne, filtrée à travers une grille d'analyse thomiste. Freud en aurait frémi... Même si les programmes de formation et les milieux de stages ne se déclaraient pas d'affiliation religieuse particulière, il n'était pas rare de constater la présence d'organismes comme le Protestant Family Agency, Jewish Family Services ou le Service catholique familial. Plusieurs de ces organismes prônaient certaines politiques vis-à-vis le divorce, l'avortement, le contrôle des naissances, qui influençaient énormément nos interventions en tant que travailleuses et travailleurs sociaux.

Reflets: Plusieurs organismes de service social ont effectivement été mis sur pied par des communautés religieuses.

R. Lecomte: En effet, ces racines religieuses étaient encore très présentes au cours des années 1950 et 1960. Par exemple, les écoles de service social des universités Laval et de Montréal ainsi que celle de St.Patrick's College, furent fondées par des communautés religieuses. Il ne faut pas se surprendre des orientations plutôt confessionnelles qui caractérisaient la formation et la pratique du service social dans les années 1940 et 1950 et même au début des années 1960.

Mais,j'ai eu mes premières expériences en service social en 1959, à la Société de l'aide à l'enfance d'Ottawa-Carleton, avant de poursuivre des études de maitrise. C'était très fréquent à l'époque d'embaucher des intervenants possédant un diplôme de premier cycle et de leur offrir une formation sur place comme «apprenti». Ce que fit un grand nombre de mes collègues. L'admission à la maîtrise requérait une expérience pratique qui était fournie d'une façon idéale à la Société de l'aide à l'enfance et qui donnait l'occasion à de nouveaux travailleurs sociaux de se familiariser avec les aspects légaux, humains et familiaux de la pratique. C'était vraiment une formation de base générique. 
Ce n'est qu'au niveau de mes stages de maitrise que j'ai connu les aspects religieux et confessionnels de la pratique. Sans élaborer davantage sur ce sujet fort litigieux, il importe de rappeler que la profession du service social a des racines religieuses et judéochrétiennes fort profondes que l'on ne doit pas ou que l'on ne peut pas oublier!

Reflets : De quelle façon cette formation a-t-elle été marquante pour vous?

R. Lecomte: Un des aspects les plus frappants était la nature dogmatique de la formation, surtout technique, qui n'invitait presque aucune analyse critique et qui tenait peu compte du contexte socio-politique de la pratique du service social. Il y avait peu de choix théorique ou de questionnement; tu étais freudien ou tu ne l'étais pas; très peu d'autres approches étaient enseignées. La terminologie était freudienne et tout l'enseignement en était imprégné. Nous, les étudiantes et les étudiants - la proportion de femmes et d'hommes, inscrite au programme, était à peu près égale — avions souvent l'impression de se faire psychanalyser par nos superviseurs. Par exemple, si tu arrivais à temps, tu étais compulsif; si tu arrivais avant le temps, tu étais névrosé; si tu arrivais en retard, tu démontrais une résistance inconsciente; tu ne pouvais pas y échapper, il y avait toujours une pathologie sous-jacente. Plus tard,j'ai constaté la même rigidité dans l'enseignement de certaines approches en service social qui se prêtaient mal à une analyse critique de la part des étudiants. J'ai alors réalisé que le dogmatisme n'appartient pas seulement à l'affiliation religieuse mais aussi à l'affiliation idéologique...

Reflets: Quelles étaient les formes d'intervention les plus répandues en service social au cours des années 1950 et 1960?

R. Lecomte: L'accent était beaucoup sur le casework mais le mot intervention ne faisait pas encore partie du vocabulaire. Le casework impliquait alors des interventions psychosociales ou, plutôt, des thérapies psychosociales pour employer le discours du temps. Le terme casework était aussi utilisé au Québec, même dans les universités francophones. Il y avait peu de cours sur le groupe, très peu d'enseignement sur l'intervention communautaire et encore moins sur les politiques sociales. Certains programmes canadiens comme 
à l'Université de Toronto, par exemple, avaient commencé à intégrer cette matière plus tôt. Mais, c'est seulement à la fin ou au milieu des années 1960 qu'on voit apparaître des axes plus résolument sociaux dans les programmes en service social. Il faut ajouter qu'il n'y avait qu'une université canadienne, celle de Toronto, qui offrait la possibilité de faire des études de troisième cycle en service social. La plupart des personnes désirant faire des études doctorales en service social devaient s'exiler aux ÉtatsUnis. J'ai donc fait mes études de doctorat aux États-Unis. Nous revenions avec un bagage américain et anglophone et nous avions été exposés à des concepts qui commençaient tout juste à faire l'objet de discussions ici, à peu près à la même époque où le service social canadien a commencé à s'ouvrir davantage aux sciences sociales. L'étude de l'apport des sciences sociales à la formation et à la pratique en service social a profondément marqué ma carrière. En fait, ma thèse de doctorat a porté sur l'analyse de la connaissance provenant des sciences sociales dans la pratique du service social, ce qui m'a amené à enseigner plusieurs cours sur les fondements théoriques et l'évaluation des pratiques sociales dans diverses écoles de service social.

Reflets: Vers le début des années 1970, l'apport des sciences sociales dans le champ du service social a soulevé des débats importants qui ont mené à des transformations de la pratique. Comment se sont manifestées ces transformations?

R. Lecomte : Les transformations ont été influencées par le contexte social des années 1970 et ont fait l'objet de réflexions et d'écrits qui tentaient de déterminer la pertinence de certains concepts des sciences sociales. Par exemple, la théorie des rôles, celle des systèmes ou encore la théorie de la communication ont permis l'établissement des fondements théoriques en service social. Certains écrits, émanant surtout de professeurs d'universités américaines, tels que Perlman de l'Université de Chicago, Hollis de l'Université de Columbia et Satir de l'École de Palo Alto, ont eu une influence prépondérante dans l'élaboration de cette ligne de pensée. Les sciences sociales offraient aussi des analyses nouvelles des problèmes humains et sociaux qui débouchaient sur d'autres types d'interventions. Par ailleurs, la question du développement 
communautaire et de l'animation sociale et politique commencent à se poser, surtout au Québec. Les tensions deviennent de plus évidentes entre les tenants des approches dites «cliniques» ou «directes» et ceux des approches dites «communautaires» et «d'action sociale». Ce dualisme entre le "personnel» et le «social», entre les soi-disant "agents de contrôle» et les soi-disant "agents de changement", a créé des querelles et des divisions énormes au sein des corps professoraux de plusieurs universités canadiennes, qui, dans certains cas, ne sont pas encore réglées.

Les enjeux identitaires du service social se posaient donc en ces termes dualistes: tu travaillais soit avec les individus et les familles, en adoptant une orientation surtout psychologique et consensuelle, soit avec des groupes communautaires et des organisations, en adoptant une orientation surtout sociologique et conflictuelle. Ces derniers intervenants étaient très minoritaires mais très vocaux... J'ai constaté le malaise que j'éprouvais face à ce dualisme lorsque j'étais à l'Université de Montréal. Avions-nous importé cette tension des sciences sociales où l'on constatait ce débat entre la psychologie et la sociologie?

Mon questionnement m'a incité à me joindre à l'École de service social de l'Université Carleton, où une équipe fort dynamique travaillait à articuler une approche "holistique» et "structurelle» qui reconnaissait l'importance de lier le «personnel» et le «social» dans la formation et dans les interventions en service social. J'ai d'ailleurs rédigé un chapitre de livre sur ce sujet, "Connecting Private Troubles and Public Issues in Social Work Education», dans lequel je tentais d'articuler un cadre théorique pour l'approche structurelle en service social.

De toute évidence, les sciences sociales ont fourni des concepts fort puissants dans l'analyse des problèmes sociaux. Les notions de changement social, de conflit, de classe, de race, d'ethnie, etc., offraient des perspectives fort stimulantes et provoquaient des débats des plus intéressants sur la nature des interventions en service social. Mais, c'est certes la question du sexisme et le féminisme qui ont soulevé le plus de débats sur la pertinence des analyses dites «patriarcales» provenant des sciences sociales et qui ont engendré des réflexions des plus stimulantes sur la nature même 
de la profession du service social.J'ai des souvenirs fort mémorables des étudiantes et étudiants inscrits au programme de maitrise dans les années 1970 et 1980. Leur enthousiasme à vouloir changer la société, la rendre plus équitable et juste, était très contagieux. Souvent, les professeurs étaient à la remorque de leurs idéaux et cela nous forçait à nous dépasser.

Reflets: Les revendications sociales qui ont marqué les années 1970, vous les avez d'abord connues dans le contexte du climat social américain : naissance du mouvement féministe, émeutes raciales et contestations contre la guerre du Vietnam, etc. De quelle façon avez-vous été marqué par ces bouleversements sociaux qui venaient sans doute choquer l'éducation plus traditionnelle que vous aviez reçue?

R. Lecomte: J'aimerais situer ma réponse dans le contexte de ma formation initiale en service social à St.Patrick's College. La formation clinique et freudienne offerte à cette institution m'a profondément marqué. La question sociale me préoccupait comme citoyen mais non comme clinicien. En fait, j'ai enseigné le cours Individual and family casework à des étudiantes et des étudiants noirs à l'Université de Wayne State de 1966 à 1968. Tous les jours, je traversais un ghetto noir pour me rendre au travail. Je constatais la pauvreté extrême qui y existait mais je ne voyais pas de liens avec mes enseignements cliniques. Ce sont les tragiques émeutes de l'été 1967 où des dizaines de Noirs furent assassinés par la police et les domiciles de mes propres étudiants incendiés qui m'ont amené à prendre conscience que je ne pouvais plus enseigner de la même façon. L'assassinat de Martin Luther King créa un désarroi majeur chez plusieurs d'entre nous. Mais, comme plusieurs de mes collègues universitaires, je me sentais impuissant face à cette conjoncture. Nos cadres d'analyse ne pouvaient expliquer cette injustice criante et ne fournissaient pas de stratégies d'intervention pertinentes. On voulait changer le «système» sans trop savoir de quoi il s'agissait! Il me manquait une analyse plus large et plus pertinente. J'ai alors décidé de retourner aux études en 1968, dans un programme doctoral qui combinait la recherche sociale et les sciences humaines appliquées, offert à Bryn Mawr College en Pennsylvanie. Ce fut un point tournant dans ma carrière. Pour un petit gars de l'Abitibi, ce fut tout un défi! 
Reflets : Vos études de troisième cycle terminées, vous êtes retourné au Québec où

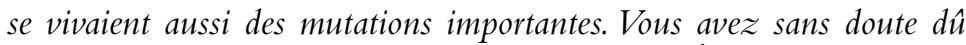
faire certaines adaptations suite à votre séjour aux États-Unis.

R. Lecomte : Pendant l'examen oral qui faisait partie des exigences du doctorat, j'avais été bouleversé par la question d'un historien qui m'avait demandé si j'avais lu Les nègres blancs d'Amérique de Vallières. J'ai dû répondre que non. C'est alors que j'ai réalisé à quel point j'avais été absorbé par le contexte américain. C'est à mon retour à Montréal, en 1971, que j'ai pu constater l'ampleur et la portée de ce qui se passait ici. L'Église s'était retirée de son rôle traditionnel face aux services sociaux et la présence de l'État se faisait de plus en plus sentir. Et ce dernier commençait aussi à définir la profession du service social. En plus des tensions entre le «personnel» et le «social» décrites précédemment, je constatais une certaine fragmentation de la profession face à cette nouvelle conjoncture. J'ai aussi été témoin d'un débat d'envergure sur les fondements mêmes de la profession qui se manifesta autour d'un intérêt croissant face à la recherche évaluative. On cherchait à vérifier l'efficacité du service social. Mais je dois ajouter que cette préoccupation provenait d'abord des instances gouvernementales, en particulier de la Commission Nepveu-Castonguay.

Un de mes premiers ouvrages: Introduction aux méthodes de recherche évaluative, a d'ailleurs porté sur cette question. Je signalais l'importance de préciser les enjeux épistémologiques, politiques et sociaux sous-jacents à l'évaluation des pratiques en service social et je mettais en évidence la pertinence des approches qualitatives, alors que les approches quantitatives étaient trop souvent privilégiées dans ce processus. Mais, il m'apparaissait évident que la profession était encore confrontée à une crise identitaire qui se définissait par un questionnement sur son efficacité, exprimé par le biais d'un discours positiviste. Est-ce que les pratiques devaient se fonder sur l'évidence, sur ce qu'on nommait evidence-based practice? Quelle place accordait-on aux aspects qualitatifs ou phénoménologiques?

On reprochait au service social de n'avoir aucune identité professionnelle ou théorique parce qu'il se fondait sur des expériences individuelles et subjectives et sur des connaissances 
empruntées aveuglément des sciences sociales. En d'autres mots, comment pouvait-on prétendre à un statut professionnel à partir d'un corpus de connaissances aussi fragile et si peu «objectif»? Combiné à ce questionnement, on retrouve le débat entre les soi-disant «agents de contrôle social», c'est-à-dire les cliniciens et les «agents de changement social», les interventionnistes communautaires auxquels j'ai fait allusion plus tôt. Dans la foulée de ce débat, ont surgi les approches structurelles et radicales, féministes et interculturelles.

Reflets: Dans vos propos, on constate à quel point le service social est remis en question à travers ces débats et on voit aussi émerger des approches nouvelles et radicalement différentes?

R. Lecomte: Le contexte socio-politique des années 1980 et 1990, le rôle changeant de l'État et la décentralisation du social ont provoqué des transformations d'envergure dans la conceptualisation et la prestation des services sociaux et de santé ainsi que dans la fonction même de la profession du service social. Une analyse critique des rapports entre l'État et la profession commence à s'articuler dans certains milieux. On s'interroge sur l'autonomie réelle de l'État et sur les motifs qui l'incitent à se désengager de ses responsabilités face au bien-être social, par exemple. Des analyses encore plus critiques apparaissent autour de la nature même des rapports sociaux et des structures sociales qui les maintiennent.J'ai participé activement, à l'École de service social de Carleton, à l'articulation d'une approche dite «structurelle» qui était fondée sur la conviction qu'il existe des liens très étroits entre les problèmes individuels et humains et les structures sociales qui sont souvent la source principale de ces problèmes. Ce positionnement a engendré plusieurs initiatives dont la mission principale était d'abord de bien comprendre cette problématique et d'agir en adoptant une orientation de changement social visant à améliorer la condition individuelle, et non l'inverse comme il était souvent prôné dans les approches dites "conventionnelles». Dans le nouveau contexte de globalisation et de rationalisation mondiale, je crois que cette analyse est des plus pertinentes. Quoi qu'en pensent certains individus, les approches axées sur le «social» n'abandonnent pas la personne, elles ne font que la situer dans un contexte plus large 
où elle n'est pas uniquement responsable de tous ses déboires et où l'empowerment lui reconnaît toute sa dignité.

Reflets : On est passé rapidement des années 1970 aux années 1980 et 1990. Lorsque vous parlez d'une situation de globalisation et de mondialisation, il est question d'une situation d'éclatement, de rupture. Comment cette nouvelle conjoncture se manifeste-t-elle au niveau du travail social?

R. Lecomte : Les années 1980 et 1990 sont caractérisées par un désengagement de l'État et la décentralisation du social et cela, au niveau mondial. L'enthousiasme axé sur la possibilité de pouvoir changer la société se bute à un genre d'impuissance et de désenchantement chez plusieurs d'entre nous. La précarité d'emploi dans les milieux "progressistes», c'est-à-dire où l'on pouvait faire des changements sociaux, est de plus en plus évidente. Les gouvernements privilégient souvent des programmes qui nécessitent moins de formation universitaire afin de couper les coûts. On constate la présence d'autres professions qui viennent faire compétition aux postes précédemment assignés aux travailleuses et travailleurs sociaux. Les approches structurelles qui étaient perçues auparavant comme étant très controversées font maintenant partie de la gamme des approches enseignées dans les écoles de service social. Nous n'avons pas à convaincre la plupart de nos étudiantes de la présence d'inégalités sociales, de pauvreté, de discrimination sexuelle et raciale.

Le problème se situe surtout au niveau de l'intervention sociale et de la possibilité de changer ces réalités. On sent de l'impuissance face à l'ampleur des problèmes humains et sociaux. De façon générale, ce n'est plus l'analyse qui fait défaut, mais plutôt l'intervention. Et paradoxalement, on constate un intérêt marqué pour les interventions dites "micro», c'est-à-dire celles au niveau des individus, des petits groupes et des familles. On revient donc à des méthodes d'intervention qu'on utilisait dans les années 1960 et 1970, mais avec une analyse beaucoup plus large qui, nous l'espérons, va influencer l'évolution des pratiques, à la longue.

Reflets: Cette analyse fait partie des programmes de formation en service social et les étudiantes y sont exposées à plusieurs niveaux. Elles possèdent souvent le langage pour discuter de la diversité, du multiculturalisme, des rapports 
sociaux de sexe, mais tout se joue comme s'il n'y avait plus ou peu de milieux de pratique où peuvent s'actualiser des approches qui s'appuient sur ces analyses-là.

R. Lecomte: Il y a cela et il y a aussi un autre phénomène que ma fille a étudié lorsqu'elle a fait sa thèse de maitrise en sociologie intitulée : That was then, this is now: understanding young women's experience and perspectives on feminism and the women's movement. Elle a interviewé des jeunes femmes, à qui elle a demandé comment elles percevaient le féminisme et comment elles concevaient leur participation dans le mouvement des femmes.

Au premier abord, ces jeunes femmes ne voulaient pas s'identifier comme féministes. L'analyse de leur discours a cependant révélé que dans leur quotidien, avec leurs amies, leurs copains, elles avaient une orientation très féministe. Mais c'est la nature de leur implication ou de leur action sociale qui est intéressante. Ces jeunes femmes, comme plusieurs des étudiantes des écoles de service social, ont connu le féminisme dans un contexte fort différent de leurs ainées. Ces dernières ont vécu la période dite «collective et globale» du mouvement alors que les jeunes femmes d'aujourd'hui connaissent une dimension dite «moléculaire» de ce mouvement. Cette dimension s'exerce par le biais d'actions concrètes, vécues quotidiennement. C'est un genre de "personal collective action». La génération de leurs ainées a peut-être créé le féminisme, mais ces jeunes femmes le vivent quotidiennement dans leurs interactions sociales.

On peut faire un parallèle avec ce qui se passe présentement en travail social: les gens ne questionnent plus l'analyse, ils questionnent plutôt les modalités de changement, d'action sociale, etc. En tant qu'éducateurs, il m'apparaît très important de comprendre cette perspective. J'ai d'ailleurs articulé cette problématique lors d'un exposé à un congrès de l'ACESS qui s'intitule L'éducation en service social vers l'an 2000 : réflexions d'un ex baby-boomer, où j'ai tenté d'identifier quelques enjeux intergénérationnels sur le plan idéologique entre les professeurs et les étudiants dans la formation en service social.

Je crois que les préoccupations de la génération actuelle d'étudiantes et d'étudiants s'expriment différemment de celles 
de ma génération et de celles de plusieurs des professeures et professeurs en service social qui sont, pour la plupart, des babyboomers et qui ont connu toute une autre gamme d'expériences. D'ailleurs, divers écrits, tel Boom, Bust and Echo de David Foot, ou Une génération bouc émissaire : enquête sur les baby-boomers de Jacques Grand'Maison, démontrent bien la complexité de la question intergénérationnelle.

Reflets : Vous avez beaucoup parlé d'éclatement, de diversité et de ruptures ce qui amène à soulever la question du courant postmoderniste qui suscite présentement beaucoup d'intérêt chez de nombreux chercheurs, notamment dans le domaine des sciences sociales. Dans une récente communication, vous avez d'ailleurs partagé certaines de vos observations au sujet de cet engouement pour la perspective postmoderniste. De quelle façon cet intérêt se manifeste-t-il dans les pratiques actuelles en service social?

R. Lecomte: Je crois qu'il existe un lien essentiel entre l'identité et un type d'orientation. Comme je le soulignais plus tôt, au cours de son histoire, notre profession a été caractérisée par un tiraillement identitaire et idéologique. La notion d'identité varie énormément selon qu'elle se rattache à des orientations philosophiques comme le modernisme ou le postmodernisme. Il faut d'abord préciser que ces deux orientations fondamentales ont plongé un nombre incroyable de disciplines, autant dans les sciences sociales que dans les humanités, dans une mise en question épistémologique et ontologique sans précédent. Le service social n'y a pas échappé.

Le courant moderniste a son origine dans la Renaissance à la fin $\mathrm{du}$ XVIII ${ }^{\mathrm{e}}$ siècle. Ce courant fut très révolutionnaire et fut même personnifié dans la Révolution française. Les croyances sousjacentes à cette orientation devaient conduire l'humanité à l'atteinte du progrès, à l'émancipation, à la libération, etc. Les valeurs universelles d'égalité, de liberté, de justice, de solidarité, de vérité, dominent le discours moderniste. La trajectoire historique de ce courant est caractérisée par la croyance que le changement est possible à travers la raison, l'ordre et la science.

La profession du service social fut et est encore profondément influencée par ce courant moderniste. Paradoxalement, les approches freudiennes, structurelles, béhavioristes et certaines approches humanistes et féministes sont ancrées dans cette 
perspective. Le débat fondamental n'est pas entre la gauche et la droite, comme le souligne si bien Peter Leonard dans son ouvrage Postmodern Welfare, car ces approches se situent essentiellement dans la perspective moderniste.Toutes ces approches ont une vision de la réalité qui se manifeste à travers des structures le plus souvent implicites, inconscientes, telles le ça, le patriarcat, les classes sociales ou le «système», qui peuvent et doivent être élucidées par des techniques de conscientisation, de sensibilisation, de transfert psychologique et ainsi de suite. Les comportements individuels et sociaux ont une logique qui peut être saisie et analysée d'une façon rationnelle et systématique. Cette perspective requiert l'analyse des causes, une classification ou une typologie (telle celle du DSM-IV) et débouche sur des interventions qui visent la résolution des problèmes. Il s'agit d'une logique de découverte plutôt que de construction de la réalité. Les intervenants doivent acquérir des expertises particulières afin de mieux saisir la réalité objective sous-jacente aux problèmes manifestés par leur clientèle. La théorie devient instrumentale et le plus souvent importée des sciences sociales. Le service social se perçoit ainsi comme une science sociale appliquée et privilégie la méthode scientifique dans sa recherche identitaire de crédibilité professionnelle.

Par ailleurs, le postmodernisme rejette la plupart des présupposés essentialistes du courant moderniste. On peut se demander s'il s'agit d'une évolution du modernisme, d'un méta-mouvement ou, tout simplement, d'un courant réactionnaire. Avec les prémisses modernistes, je crois que le désenchantement s'est manifesté en service social surtout depuis le milieu des années 1980 autour du débat sur la pertinence des méthodes de recherche quantitatives et qualitatives. Certains soutenaient que la crédibilité professionnelle du service social serait accrue si son corpus de connaissances était davantage axé sur la recherche empirique, quantitative et objective. Le courant postmoderniste se définit en termes de pluralisme, d'éclectisme, d'écologisme culturel et met davantage l'accent sur la différence et la diversité que sur la recherche de qualités communes et universelles. Les approches constructivistes, qui jouissent d'une popularité croissante en service social, ont une grande affinité avec ce courant. 
Depuis le début des années 1990, on constate une sorte d'engouement pour la perspective postmoderniste en service social. Mais quelques auteurs, quoique très ouverts à ce courant, invitent les travailleuses et travailleurs sociaux à manifester une certaine prudence à ce sujet. La relation ambivalente du féminisme au postmodernisme est très révélatrice à cet égard. Par exemple, la catégorie universelle «femme» doit-elle être déconstruite afin de permettre l'analyse des oppressions multiples et différentes des femmes à travers le monde? Cela remet en question plusieurs notions de solidarité et de projets émancipatoires universels en faveur d'approches plus locales, microscopiques et ponctuelles.

Je me réjouis de la critique postmoderniste à l'égard du dogmatisme et de la rigidité de certaines approches modernistes. Cependant,je m'inquiète d'un certain relativisme philosophique, un "relativisme doux ", comme le décrit si bien Charles Taylor, qui met davantage l'accent sur l'individu, son épanouissement, etc., en banalisant le rôle du social. Les valeurs universelles de justice sociale, de solidarité, ont-elles encore une place? Je le crois.

Reflets : Si on se dirige vers plus de précisions dans l'analyse sociale, est-ce qu'il n'existe pas le danger que l'on se dirige vers une autre forme de positivisme?

R. Lecomte: Le courant postmoderniste nous fait comprendre que notre discours et le langage que nous utilisons, ne sont pas seulement un reflet d'une réalité objective comme le soutiennent les tenants de l'approche moderniste, ils sont aussi une construction sociale. Nous construisons notre réalité à travers notre langage, nous sommes changés par l'autre en l'écoutant. Cela a forcément des implications majeures pour nos pratiques sociales. Nous ne sommes pas des observateurs objectifs et nos clients ne sont pas des sujets passifs qui doivent être étudiés et classifiés selon une grille d'évaluation ou une typologie de catégories préétablies. Les clients ne sont pas consultés dans un plan d'intervention déjà établi, mais ce sont eux qui fournissent le contexte interprétatif qui détermine la nature du problème. Le monde du client est conçu comme une toile de significations, tissée et soutenue au travers du langage. Cette stratégie rompt avec les dilemmes où s'opposent sujet et réalité, subjectivité et objectivité. 
Si l'on considère le positivisme comme une doctrine qui se réclame de la seule connaissance des faits et de l'expérience scientifique, le «logico-positivisme», je ne crois pas que l'orientation que je viens de décrire se situe dans cette perspective. Elle se situe davantage dans la tradition du verstehen (comprendre par empathie) prônée par Max Weber.

Reflets: En vous écoutant, on constate que, d'une certaine façon, c'est très libérateur pour l'intervenant, alors que de l'autre, c'est aussi très insécurisant : on travaille un peu dans l'inconnu, on doit être prêt à prendre des risques.

R. Lecomte : J'ai enseigné le cours de «Fondements théoriques du service social» pendant au-delà de 20 ans. La première phrase que je disais en classe et que je répétais au dernier cours, c'est que les travailleuses sociales et les travailleurs sociaux œuvrent dans un contexte d'incertitude, avec des problèmes dont on connait très peu la cause; de plus, ils ou elles ne pourront jamais démontrer de façon scientifique qu'ils peuvent changer seuls les conditions qui ont occasionné ces problèmes. La pauvreté, la maladie mentale, etc., sont des problèmes complexes; les travailleuses sociales et les travailleurs sociaux qui veulent œuvrer auprès des victimes de ces conditions doivent faire preuve d'une grande humilité et d'une grande créativité.

Certaines approches ont malheureusement prôné une analyse très superficielle de ces problèmes et ont souvent blâmé les «victimes» comme étant les responsables de leur condition. On n'aime pas travailler dans un contexte d'incertitude, surtout lorsqu'on veut être un professionnel crédible, c'est-à-dire, scientifique. Mais voilà, on travaille avec une variété de problèmes dont la cause est fort controversée. Par exemple, on a qu'à penser à la schizophrénie, au sujet de laquelle de nombreux schèmes explicatifs sont couramment offerts : de la structure de la personnalité à la structure des communications familiales, aux prédispositions héréditaires et biologiques, ainsi de suite. La travailleuse sociale doit et peut agir dans ce contexte d'incertitude scientifique en ne se situant pas dans un schème de référence linéaire ou de cause à effet caractéristique du courant moderniste. Je reconnais que cela n'est pas facile dans le contexte actuel de reconnaissance professionnelle fondé sur evidence-based practice. On recherche «l'aura» rassurante 
de l'expert, fondée sur une notion équivoque de la science qui est loin d'être unanime lorsqu'il s'agit d'expliquer les causes des problèmes humains et sociaux. C'est ça le malaise du modernisme, c'est qu'on est mal à l'aise avec l'incertitude. Pourtant, c'est la vie... N'est-ce pas?

Reflets : Il s'agit donc d'un courant qui suscite des approches qui peuvent devenir très individualistes. Est-ce qu'il n'y a pas un danger de tout recentrer sur l'individu?

R. Lecomte : C'est une très bonne observation, parce que la première critique qu'il faut faire du postmodernisme, c'est qu'il affiche une certaine tendance à relativiser les problèmes sociaux et humains en se fiant surtout aux perceptions subjectives et intersubjectives de ces problèmes. Comme le disait ce praticien aux allures «postmodernistes» : "Ça dépend...». Le problème, c'est qu'en relativisant tout, on risque d'occulter l'absolu, les valeurs universelles de solidarité, de justice sociale, ainsi de suite. Comme tout est relatif, ça dépend de l'histoire de la personne, de sa perspective, etc. Il est intéressant de noter que plusieurs auteurs américains et canadiens qui adoptent cette orientation, proposent davantage des interventions sur le plan individuel et psychologique qui accordent beaucoup d'attention aux significations accordées au vécu des problèmes humains. Dans les approches constructivistes, par exemple, on a trop souvent tendance à situer l'intervention au niveau intersubjectif et, paradoxalement, d'occulter le "social» des interventions. D'ailleurs, certains tenants du postmodernisme se sont vite empressés de se servir de leur analyse pour critiquer la pertinence même du féminisme comme mouvement social.

Le débat est mal engagé s'il polarise à nouveau le privé et le public, le qualitatif et le quantitatif et ainsi de suite. N'y a-t-il pas une réalité objective de la pauvreté croissante, de l'oppression des femmes, au niveau mondial, qui risque de devenir une «culture de la pauvreté» ou un problème de perception, si l'on se limite aux seuls facteurs intersubjectifs de cette réalité?

Par contre, on sait que le mouvement féministe contemporain a réfuté ce dualisme, entre le politique et le personnel, notamment. Il faut revenir à cette dialectique entre les deux dans les pratiques contemporaines. On ne peut pas dire qu'une personne a un 
problème sans comprendre les liens entre celui-ci et les contextes socio-politique et économique. Il faut faire l'articulation entre les deux. Or, avec l'effritement et l'éclatement de nos pratiques et politiques sociales, nous avons été en quelque sorte forcés d'opter pour l'une ou l'autre de ces perspectives. Opter pour des pratiques dialectiques crée une tension, un tiraillement, mais je me dis que c'est ça la pratique du service social.

Reflets : Suite à cette analyse de l'évolution du travail social depuis quarante ans, comment le définiriez-vous aujourd'hui?

R. Lecomte : On ne peut comprendre le travail social qu'en le situant dans les contextes économique, politique et idéologique où il a pris forme et en tenant compte des rapports et des enjeux sociaux qui se sont développés dans la société. Le travail social est constamment «travaillé» dans ses orientations et ses définitions, et c'est cela qui le rend si intéressant et si dynamique. Les diverses définitions se situent autour de débats fondamentaux concernant les notions de travail social comme une profession, de travail social comme un art ou une science, de travail social axé sur le fonctionnement social des individus, de travail social comme une discipline pratique axée sur le changement social. Ces discours sur la nature du travail social mettent en évidence certaines tensions dans la conception même du travail social : des tensions épistémologiques, c'est-àdire, relatives aux fondements théoriques de la profession; des tensions idéologiques, c'est-à-dire, relatives à la fonction et à l'objet même de la profession vis-à-vis son rôle comme intermédiaire et médiateur et celui d'agent de changement social.

L'étude des diverses définitions du travail social révèle donc une interrogation constante sur ses fondements, sa fonction et son objet. On ne peut qu'être frappé par la présence de diverses conceptions du travail social aux divers moments de son histoire. Ainsi, ce qui définissait le travail social à une période donnée est mis en question à la période suivante.

Je pense qu'une définition complète du travail social doit tenir compte des dimensions épistémologique, artistique et scientifique qui caractérisent la profession, de la mission originale du travail social qui vise prioritairement les couches sociales les plus défavorisées, du souci de comprendre l'individu dans son contexte 
social, de la diversité des interventions et des lieux de pratique et du contexte organisationnel où il se situe. L'orientation théorique ou idéologique privilégiée doit fournir une explication cohérente de chacune de ces composantes dans son désir de définir le travail social. Il est vrai que chacune d'elles porte des tensions internes qui occasionneront encore des débats importants sur les orientations à privilégier dans l'articulation de la nature même du travail social. C'est d'ailleurs ce tiraillement épistémologique et idéologique qui est la caractéristique majeure du travail social et qui constitue sa force et son dynamisme. Cette caractéristique ouvre la porte à une certaine démocratisation dans la définition de son objet et de sa fonction, incluant la perspective des usagers, celle des étudiantes et des étudiants, des praticiennes et des praticiens, des chercheures et des chercheurs et de tous les autres. L'identité professionnelle est enracinée dans ce contexte de tiraillement dialectique quotidien de nos pratiques sociales, ce refus de polariser le «personnel» et le «social». C'est le défi de construire notre avenir et notre identité avec l'autre... 